\title{
Erratum to: Effect of temporal spacing between advertising exposures: Evidence from online field experiments
}

\author{
Navdeep S. Sahni ${ }^{1}$
}

Published online: 26 September 2015

(C) Springer Science+Business Media New York 2015

\section{Erratum to: Quant Mark Econ DOI 10.1007/s11129-015-9159-9}

The author would like to note an error in Figure 5 on page 19 of this paper. The error is in the top-left panel of the figure. The corrected Figure is shown below. The difference between the figure in the paper and the correct one is not statistically significant. Further, the error does not change the statistical inferences drawn from the figure. This error was made while producing this figure and does not carry over to any other part of the paper.

This figure shows the mean chance of visiting the experimental advertiser's page in sessions 2 ( Visit $_{2}$ ) and 3 ( Visit $_{3}$ ), split by the time gap between the first two sessions $\left(\right.$ days $\left._{1-2}\right)$. The sample is further grouped by the number of experimental ad exposures an individual gets in session $2\left(n E x p_{2}\right)$. The bars on the top-left in Fig. 5

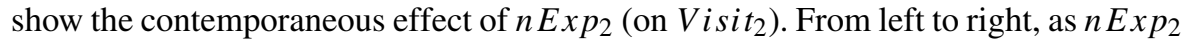
increases, the mean $\mathrm{Visit}_{2}$ increases from about $1.5 \%$ when $n E x p_{2}=0$, to about $5 \%$ when $n \operatorname{Exp}_{2} \geq 3$. This increase is statistically significant. The bars on the top-right show that such effect occurs irrespective of the spacing $\left(\right.$ days $\left._{1-2}\right)$ between the first two sessions. The graphs in the bottom row of the figure show the carryover effects of $n E \operatorname{Exp}_{2}$, the corresponding probabilities of visit in session 3. A comparison of the bars on the top-left and the bottom-left shows that the effect of increasing $n \operatorname{Exp}_{2}$ decays from the second to the third session. When $n \operatorname{Exp}_{2} \geq 3$ and days $s_{1-2} \leq 7$, the chance of a visit decreases from $5 \%$ in session 2 , to $2 \%$ in session 3 (p-val<0.05).

The online version of the original article can be found at http://dx.doi.org/10.1007/s11129-015-9159-9.

Navdeep S. Sahni

nsahni1@stanford.edu

1 Graduate School of Business, Stanford University, Stanford, CA, USA 

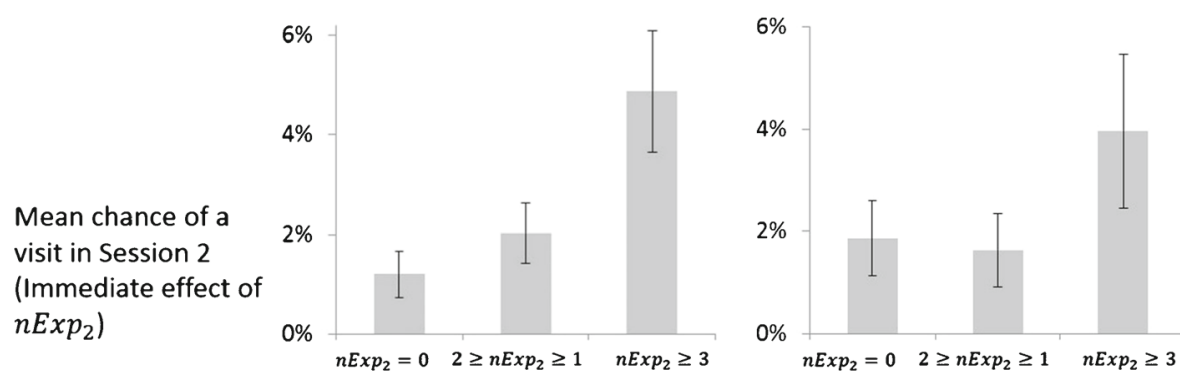

Mean chance of a visit in Session 3 (Carryover effect of $\left.n E x p_{2}\right)$

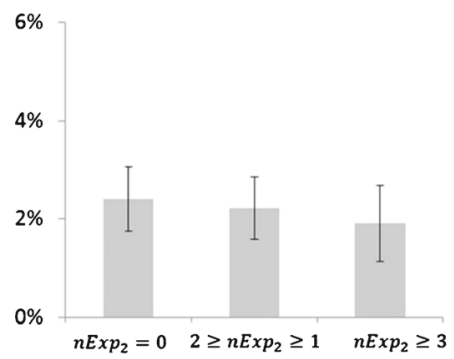

First two sessions close to each other $\left(\right.$ days $\left._{1-2} \leq 7\right)$

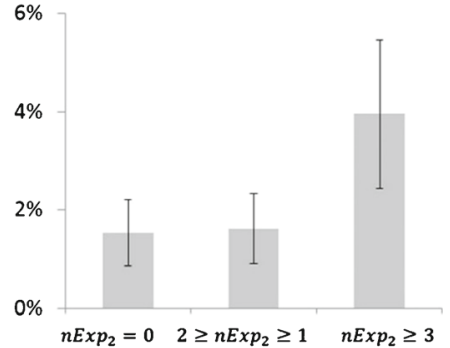

First two sessions further apart $\left(\right.$ days $\left._{1-2}>7\right)$

Fig. 5 Mean chance of visiting the advertised restaurant's page in the second and the third sessions, split by time gap between the first two sessions (days $s_{1-2}$ ), and the number of exposures to the experimental ad in the second session. The error bars show $90 \%$ confidence intervals for the means

However, (comparing bars on the top-right and bottom-right) when $n E x p_{2} \geq 3$ and days $_{1-2}>7$, there is no significant drop; the probability of a visit remains around $4 \%$ in both the sessions. 